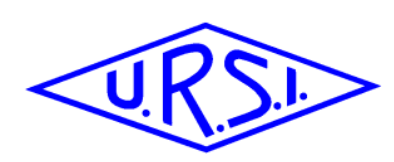

\title{
Detection of UHF RFID tags in Metallic Guided Structures
}

\author{
A. Michel ${ }^{(1)}$, M. Rodriguez Pino ${ }^{(2)}$, and P. Nepa ${ }^{(1)}$ \\ (1) Dept. Information Engineering, University of Pisa, Pisa, Italy \\ (2) Dept. Ingenieria Electrica, Universidad de Oviedo, Gijon, Spain
}

\begin{abstract}
The detection of UHF RFID tags placed inside particular structures that can approximated by rectangular or circular waveguides is here discussed. Preliminary theoretical, numerical and experimental analyses are described, relating the tag detection to the propagation of TE or TM modes.
\end{abstract}

\section{Introduction}

In recent years, Radio Frequency Identification (RFID) systems have been largely used for logistic, retail and pharmaceutical applications [1]. In general, RFID systems can be subdivided into two main groups, on the basis of the operative frequency band: High Frequency (HF, $13.56 \mathrm{MHz}$ ) and Ultra High Frequency (UHF, 865-928 $\mathrm{MHz})$ RFID bands. HF RFID systems are employed for short-range applications (e.g. proximity identification and payments) and they are based on inductive coupling between two coils. On the other hand, UHF RFID systems are used for both near-field [2], [3] and far-field applications such as localization [4], sensing and Item Level Tagging (ILT) applications.

In a number of scientific contributions, research is devoted to theoretically model the RFID signal propagation, aiming at predicting the tag detection by means of numerical results [5]. However, it is worth mentioning that, in some specific scenarios such as near-field systems, the ray model [2] typically used for long-range UHF RFID systems can not be applied, since the main requirements are not satisfied. Thus, more accurate models have been studied [5]-[7].

A possible scenario in which tag detection requires a specific model consists of tags placed inside closed structures that can be approximated with rectangular or cylindrical waveguides. For example, as shown in (Figure 1 ), ventilation and sewage pipe may be modeled with metallic waveguides [8]. On-metal tags can be attached to the internal part of the pipe, and used for identification. The issue of detecting RFID tags inside guided structures have been already addressed in the past [9]-[11]. Specifically, in [9] a full-wave electromagnetic simulation and analysis of UHF RFID read/write antenna exciting the IC tag placed inside a coaxial metal pipe is presented. Moreover, in [10] the theory of UHF RFID signal propagation within metal circular pipes has been considered. The operating limitations of passive RFID system in hollow metal pipes are identified by means of parameters such as the propagation constants, the different TE and TM modes, cut-off frequencies and the multimode attenuation. In [11], this approach has been applied to helical and toroidal structures.

In this paper, preliminary theoretical considerations are discussed to model the detection of a tag placed inside a metal pipe. Numerical and measured results are shown by considering a commercial dipole-like tag. The aim of this paper is to discuss preliminary considerations suitable to create a more accurate coupling model for UHF RFID tags inside guided structures.

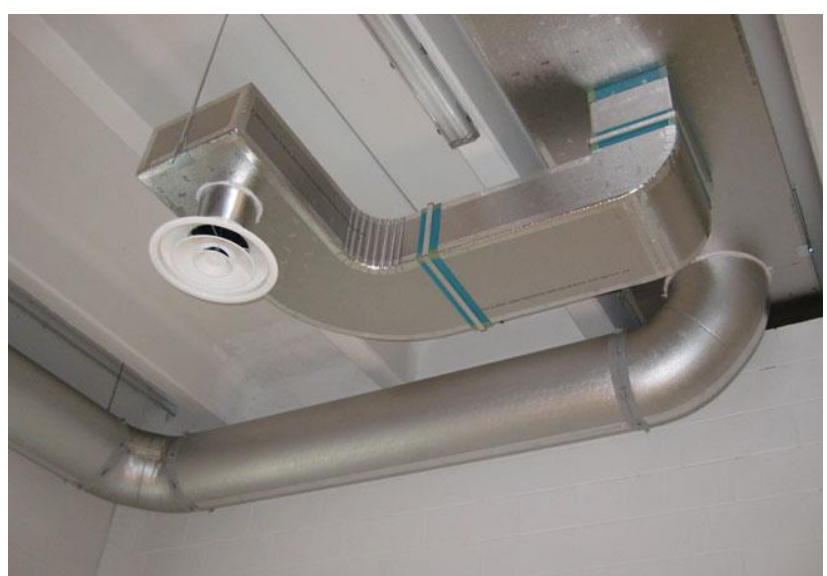

Figure 1. Example of circular and rectangular metal structures used as ventilation pipes.

\section{Layout}

In Figure 2, an example of rectangular and circular waveguides is shown, where an Inlay UH100 (LAB-ID) tag is placed inside. The EM propagation through the above structures have been analyzed by using a commercial electromagnetic software tool, namely CST Microwave Studio. The electric field distribution at the input port is also represented. In both cases, an operating frequency of 866.5 $\mathrm{MHz}$ has been considered, which is the central frequency of the ETSI UHF RFID band (865-868 MHz). The waveguide sizes are such that only the fundamental mode can propagate inside the structure, namely the $\mathrm{TE}_{10}$ 
in a rectangular waveguide (Figure $2 \mathrm{a}$ ) and a $\mathrm{TE}_{11}$ in a circular waveguide (Figure $2 \mathrm{~b}$ ).
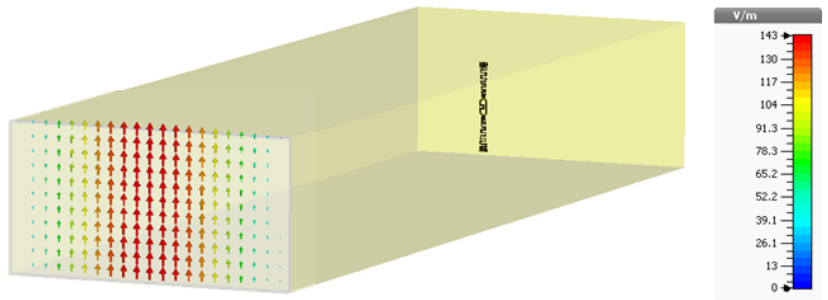

(a)
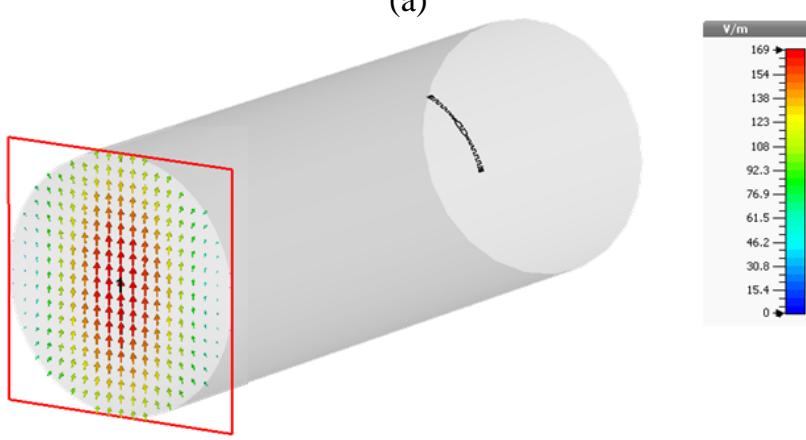

(b)

Figure 2. Approximated pipe model used for the numerical analysis: (a) rectangular and (b) circular waveguide.

For example, let us consider a metallic circular pipe approximated by a cylindrical structure with radius $a$ and length $L$. The value of the cylinder radius, $a$, is a key parameter as it is strictly related to the number of propagating modes inside the guided structure. The propagation of the fundamental mode $\left(\mathrm{TE}_{11}\right)$ in a cylindrical waveguide at ETSI UHF RFID band is allowed if the value of the radius $a$ is between $101 \mathrm{~mm}$ and $132 \mathrm{~mm}$. The electric field distribution associated to the fundamental mode is depicted in Figure 2b. For higher values of $a$, at a fixed operating frequency band, higher order modes can propagate in the cylindrical structure, so changing the electric and field distribution on the transversal plane (Figure 3).

As discussed in [3], for dipole-like tags, the electric field distribution is a key-parameter suitable to predict the RFID chip activation and tag detection. To demonstrate that the power collected by a dipole-like UHF RFID tag is proportional to the electric field distribution inside a waveguide, the cylindrical structure has been terminated on a shorting metallic plate, thus obtaining a stationary wave distribution along the longitudinal axis of the waveguide. In Figure 4, the simulated coupling ( $S_{12}$ parameter) between an UH100 Inlay (LabID) tag model and the fundamental mode $\left(\mathrm{TE}_{11}\right)$ is shown as a function of the distance of the tag antenna from the short-circuit termination. It is worth noting that a typical stationary distribution is obtained. Moreover, the distance between two consecutive minima of the tag-mode coupling is near to $30 \mathrm{~cm}$, which is close to half the guided wavelength of the fundamental mode propagating in the pipe. In the same picture, measured results are indicated by circular markers. In particular, the RSSI values have been collected while increasing the distance between tag and short circuit. An
Impinj Speedway R420 reader has been used during the measurement campaign. As expected, the normalized measured RSSI curve and the simulated coupling parameter are in a good agreement. Once again, this confirms that the signal propagation inside metal structures can be used to create an accurate theoretical model of the detection of UHF RFID tags when placed inside those objects approximated by rectangular or circular waveguides and coaxial cables.

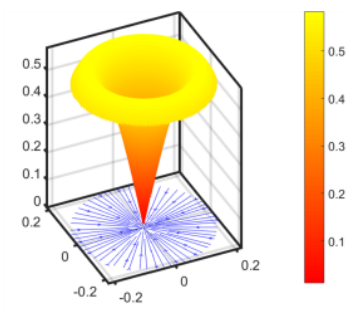

(a)

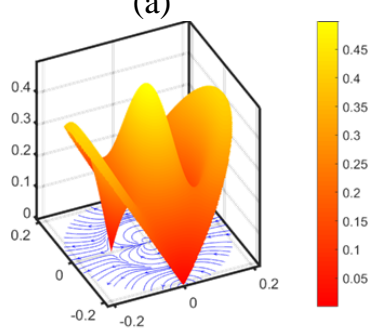

(c)

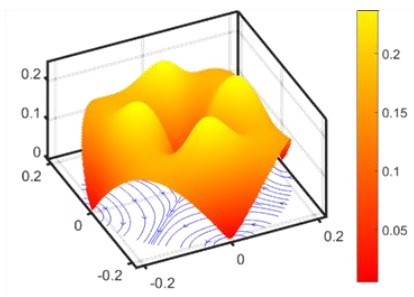

(b) (d)

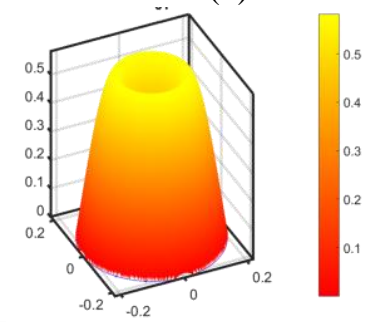

Figure 3. 3D electric field distribution of the higher modes in a circular waveguide: (a) $\mathrm{TM}_{01}$, (b) $\mathrm{TE}_{21}$, (c) $\mathrm{TM}_{11}$ and (d) $\mathrm{TE}_{01}$.

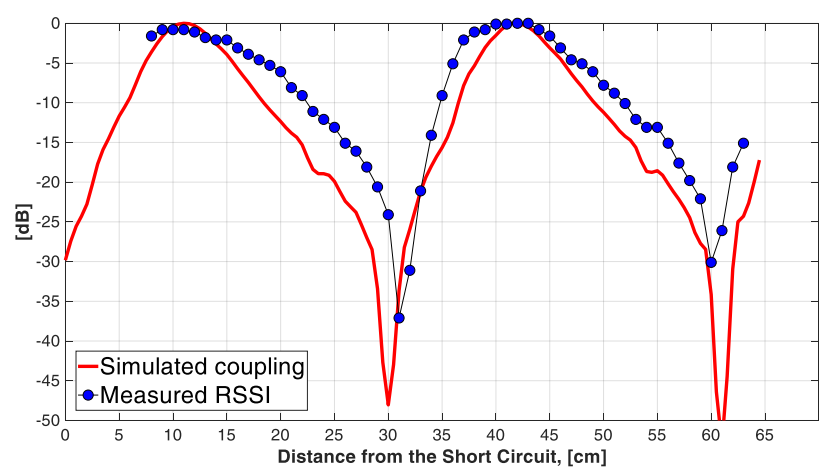

Figure 4. Simulated coupling between the fundamental mode and the dipole-like tag (solid red curve) and measured RSSI (blue markers) as a function of the distance of the tag from the shorting termination. Both the curves are normalized.

\section{Acknowledgement}

This work was supported in part by the Ministerio de Educación, Cultura y Deporte - Programa de Movilidad Salvador de Madariaga (Ref. PRX17/00635); by the Ministerio de Economía y Competitividad, under projects TEC2014-54005-P (MIRIIEM) and TEC2017-86619-R (ARTEINE). 


\section{References}

1. K. Finkenzeller, "RFID Handbook: Fundamentals and Applications in Contactless Smart Cards and Identification", 2nd ed. Hoboken, NJ: Wiley, 2003.

2. A. Michel, P. Nepa, X. Qing and Z.-N. Chen, "Considering High-Performance Near-Field Reader Antennas: Comparisons of Proposed Antenna Layouts for Ultrahigh-Frequency Near-Field Radio-Frequency Identification", to appear on IEEE Antennas and Propagation Magazine, doi: 10.1109/MAP.2017.2774141

3. A. Michel, M. Rodriguez-Pino, and P. Nepa, "Reconfigurable modular antenna for near-field UHF RFID smart point readers," IEEE Trans. Antennas Propag., vol. 65, no. 2, pp. 1-9, Feb. 2017, doi: 10.1109/TAP.2016.2640140

4. A. Buffi, M. R. Pino and P. Nepa, "Experimental Validation of a SAR-Based RFID Localization Technique Exploiting an Automated Handling System," IEEE Antennas and Wireless Propagation Letters, vol. 16, pp. 2795-2798, 2017. doi: 10.1109/LAWP.2017.2747216

5. A. Michel and P. Nepa, "UHF-RFID desktop reader antennas: Performance analysis in the near-field region," IEEE Antennas and Wireless Propagation Letters, vol. 15, pp. 1430-1433, Dec. 2015, doi: 10.1109/LAWP.2015.2511926

6. A. Michel, A. Buffi, P. Nepa and G. Manara, "Antennas for UHF-RFID printer-encoders," 2015 IEEE 15th Mediterranean Microwave Symposium (MMS), Lecce, 2015, pp. 1-4. doi: 10.1109/MMS.2015.7375399

7. A. Buffi, A. Michel, P. Nepa, and G. Manara, "Numerical analysis of wireless power transfer in nearfield UHF-RFID systems" Wireless Power Transfer, 1-12., 2017; doi:10.1017/wpt.2017.16

8. P. V. Nikitin, D. D. Stancil, A. G. Cepni, O. K. Tonguz, A. E. Xhafa and D. Brodtkorb, "Propagation model for the HVAC duct as a communication channel," in IEEE Transactions on Antennas and Propagation, vol. 51, no. 5, pp. 945-951, May 2003. doi: 10.1109/TAP.2003.811491

9. A.S. Andrenko. "EM modeling of Metal Pipe Based UHF RFID Tags Activated by R/W Antenna”, 2012 International Conference on Electromagnetics in Advanced Applications - ICEEA. Cape Town, South Africa. DOI: 10.1109/ICEAA.2012.6328630

10. D.D. Arumugam and D.W. Engels, 'Characterisation of RF Propagation in Metal Pipes for Passive RFID Systems', Int. J. Radio Frequency Identification Technology and Applications, Vol. 1, No. 3, pp. 303-343. (2007)

11. D. D. Arumugam and D. W. Engels, "Characterization of RF Propagation in Helical and Toroidal Metal Pipes for
Passive RFID Systems," 2008 IEEE International Conference on RFID, Las Vegas, NV, 2008, pp. 269-276. doi: 10.1109/RFID.2008.4519364 